\title{
Predicting Responses to Mechanical Ventilation for Preterm Infants with Acute Respiratory Illness using Artificial Neural Networks
}

\author{
Katharine Brigham*1 | Samir Gupta ${ }^{2}$ | John Brigham ${ }^{1}$
}

\footnotetext{
${ }^{1}$ Department of Engineering, Durham University, Durham, United Kingdom

${ }^{2}$ Neonatal Unit, University Hospital of North

Tees, Stockton on Tees, United Kingdom
}

Correspondence

Email: katharine.brigham@durham.ac.uk

\begin{abstract}
Summary
Infants born prematurely are particularly susceptible to respiratory illness due to underdeveloped lungs, which can often result in fatality. Preterm infants in acute stages of respiratory illness typically require mechanical ventilation assistance, and the efficacy of the type of mechanical ventilation and its delivery has been the subject of a number clinical studies. With recent advances in machine learning approaches, particularly deep learning, it may be possible to estimate future responses to mechanical ventilation in real-time, based on ventilation monitoring up to the point of analysis. In this work, recurrent neural networks are proposed for predicting future ventilation parameters due to the highly nonlinear behavior of the ventilation measures of interest and the ability of recurrent neural networks to model complex nonlinear functions. The resulting application of this particular class of neural networks shows promise in its ability to predict future responses for different ventilation modes. Towards improving care and treatment of preterm newborns, further development of this prediction process for ventilation could potentially aid in important clinical decisions or studies to improve preterm infant health.
\end{abstract}

KEYWORDS:

neonatal, time series, prediction, neural networks, deep learning

\section{1 | INTRODUCTION}

Mechanical ventilation, among other simultaneous therapies, is often necessary for preterm newborns with respiratory maladaptation, including respiratory distress syndrome and of course respiratory failure. Moreover, respiratory distress syndrome is a leading cause of morbidity and mortality in infants born prematurely with underdeveloped lungs [1]. As such, it is of paramount importance to understand and monitor the efficacy of ongoing mechanical ventilation of preterm newborns. Complicating this challenge further is that there are also questions as to how best to deliver mechanical ventilation, such as whether volume-controlled or more traditional time-cycled, pressure-limited ventilation is utilized. Towards improving care and treatment of preterm newborns, the fundamental hypothesis of the present work is that a method to predict the progression of ongoing mechanical ventilation could substantially aid in the clinical decision making and lead to increased likelihood of positive outcomes. In particular, the present study proposes the use of machine learning tools to estimate future ventilation parameters in real-time based on ventilation monitoring up to the point of analysis. 
Machine learning continues to increase in prevalence in a wide range of healthcare applications, from cancer prediction [2], to tumor volume determination using medical imaging [3], to building automated discovery and clinical decision support systems for optimising patient treatment [4]. There have also been several efforts to utilize machine learning for improving neonatal care [5], [6] . Shirwaikar et al. [7] used machine learning classification methods, such as SVMs, decision trees, and random forest to predict apnea episodes in 229 neonates during their first week of life. In this study they were able to achieve an accuracy of $88 \%$ to detect the presence of apnea using 22 input features, including heart rate at different times, gestation age, birth mode, birth weight, and others. Mikhno et al. [8] used machine learning to classify whether or not there will be extubation failure during ventilation using 6 features (e.g., heart rate) to predict the outcome. Precup et al. [9] employed SVMs to determine the combination of measures of cardiorespiratory variability, computed automatically, that best predicts extubation readiness. Their results suggest that the addition of their classification approach to current clinical measures may potentially reduce the extubation failure rate by more than $80 \%$, where about $25 \%$ of extubated infants will fail and require re-intubation. Ganzert et al. [10] proposed the use of inductive machine learning to model pressure-volume curves in artificially ventilated patients suffering from the adult respiratory distress syndrome, in that the authors sought to predict the volume given the pressure, the measurement method and the patient data. The features used included measures such as gender, age, ventilation tube type, duration, etc., for input into the CUBIST tool [11], which is an extension of a tree-based regression model with additional rules. The authors also noted that the inclusion of background information on the patient improved the accuracy of the models by almost one order of magnitude.

The aim of this study is to investigate the usage of the machine learning tools presented herein to ideally minimize future needs to test different devices on the patient in order to achieve optimal results. It is further envisioned that these tools will also provide the capability to characterize the response of a preterm infant to different types of mechanical ventilation and thus give additional insight into the basis of ventilation responses. This work is considered to be a starting point to these end goals by investigating whether or not such predictions may even be feasible. To accomplish this, there are a number of time series prediction methods that can be used, including Kalman filters, autoregressive models, linear prediction, regression analysis, and machine learning approaches. Given the highly nonlinear nature of the ventilation data, it was deemed that machine learning methods may be the most applicable for prediction as other methods tend to rely on assumptions (e.g., approximate linearity, gaussian noise) that may be inappropriate for this type of data.

Of the many machine learning methods available, ANNs in particular have gained significant popularity in the recent years due to advances in training approaches, yielding state-of-the-art performance levels in a variety of applications. One of the desirable properties of ANNs is that they are universal function approximators, so they can be used to model any arbitrary continuous function only providing that a sufficient number of ANN parameters are available [12]. ANNs are also particularly appealing conceptually as they are designed to mimic the way we perceive the human brain to function: as a network of neurons that can process information to learn patterns and concepts over time, which is an activity that the human brain is considered to excel at. There are many different variants of ANNs based on their network topology and operations and can be used for classification, regression, and prediction. In this work, we are naturally interested in the class of neural networks that can be used for time series prediction as applied to ventilation data, as their ability to model complex nonlinear functions is very desirable for this effort.

The remaining sections are organized as follows. Section 2 provides a description of the ventilation parameter dataset used in this work, and Section 3 details the type of ANN selected to predict the data. The predictions with the data are shown in Section 4. followed by a discussion of these results. Finally, concluding remarks on the findings and future work are given in Section 5

\section{2 | DATA COLLECTION}

Ventilation data was collected and examined under two types of ventilation: 1) Time-cycled, pressure-limited ventilation (TCPLV); and 2) volume-controlled ventilation (VCV). TCPLV is designed to deliver a volume of gas with a preset peak inspiratory pressure during a defined cycle time. Contrastively, VCV is designed to deliver a targeted tidal volume, and inspiratory pressure is automatically adjusted breath-to-breath in response to changes in pulmonary compliance. This data was collected in order to compare the minute ventilation between VCV and TCPLV using both assist/control (A/C) and synchronized intermittent mandatory ventilation (SIMV) modes. Pulmonary mechanics data was collected for 30 minute epochs with a washout period of 15 minutes between the epochs during the four modes of ventilation: VCV-AC, TCPLV-AC, VCV-SIMV and TCPLV-SIMV using a standardized protocol and randomized crossover design. More specifically, the ventilation protocol was randomized to start with either Volume A/C or TCPL A/C mode, and subsequent modes following the start are shown in Figure 1 


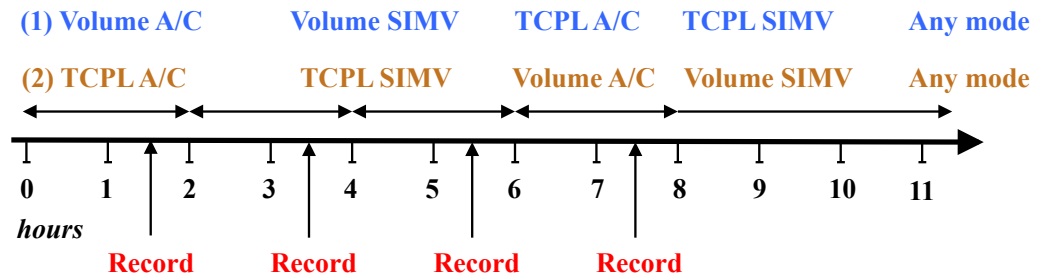

FIGURE 1 Ventilation protocol.

Breath-to-breath data for tidal volume, peak pressure and minute ventilation were collected from 10 ventilated babies of $<30$ weeks' gestation during their acute stages of respiratory illness. This included inspired and expired tidal volume, spontaneous minute ventilation and total respiratory rate, $\mathrm{FiO} 2$, pressure, and dynamic lung compliance. During each epoch, the volume (in VCV) or pressure (in TCPLV) was adjusted to provide an expired tidal volume (VTe) of 4-6 mL/kg. The control ventilator rate was maintained at 40 breaths per minute, with the inspiratory time targeted between 0.25 and 0.35 seconds. A 10 minute period of artefact-free data was used for analysis from each epoch. Measurements were taken every 1.5 seconds.

\section{3 | ARTIFICIAL NEURAL NETWORKS}

For ventilation parameter predictions, a variant of the Artificial Neural Network (ANN) will be used. The most basic type of ANN is a feedforward neural network. The architecture of a three-layer feedforward neural network is shown in Figure 2 and consists of an input layer, a hidden layer, and an output layer, interconnected by weights (to be determined), which are represented by arrows between the layers. There is also a bias unit that is connected to each node in addition to the input nodes, and at each hidden node $a_{1}, \ldots, a_{L}$ there is an activation function applied to the hidden node inputs, which is typically a nondecreasing, bounded function such as the sigmoid.

\section{1 | Recurrent Neural Networks}

The recurrrent neural network (RNN) differs from the conventional feedforward neural network in that it has a feedback mechanism where outputs are subsequently used as inputs in the following time step. It can be thought of as an extension of the basic feedforward neural network to a deep (i.e., multi-layered) feedforward neural network. An excellent illustration of the RNN architecture is given in [13] and is shown here for convenience in Figure 3. where $x_{t}$ is the input at time $t, h$ is the hidden layer (note that the weights are shared across time), and $\hat{y}_{t}$ is the RNN output at time $t$. Note that in this deep feedforward neural network, each layer is a time step. The RNN is generally considered to be more powerful than conventional neural networks due to its ability to theoretically represent richer, more complex models and sequences. However, in practice, the RNN had gained

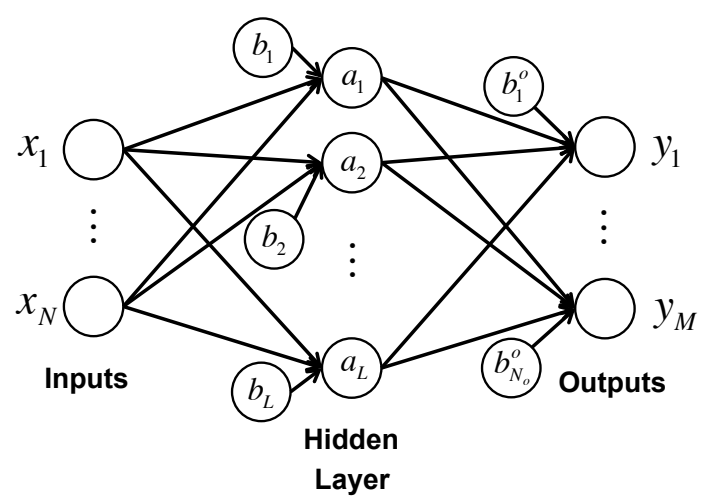

FIGURE 2 Basic feedforward ANN architecture with time-lagged inputs. 


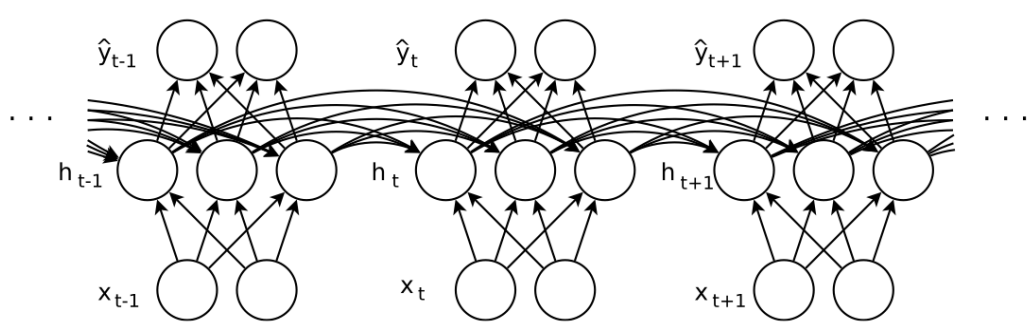

FIGURE 3 Conceptualization of the recurrent neural network architecture (from [13]).

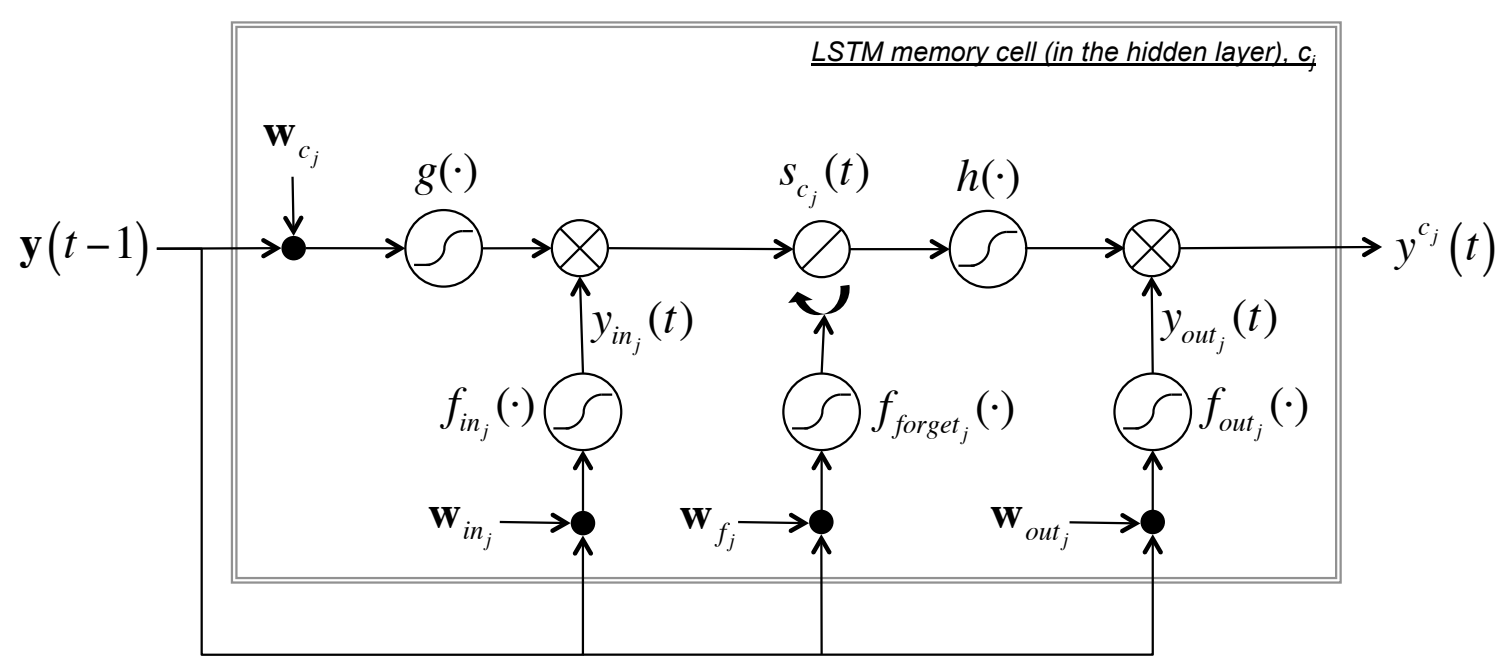

FIGURE 4 Architecture for a single memory cell in an LSTM network (in the hidden layer(s)).

a notorious reputation of being difficult to train properly. As backpropagation is the most common approach to training neural networks (i.e., learning the appropriate ANN parameters), prominent methods used for training the RNN were historically either Back-Propagation Through Time [14] or Real-Time Recurrent Learning [15], which are both gradient-based approaches. These methods would often fail to train an RNN even for relatively simplistic sequences, especially as the temporal span of the inputs increased [16]. In [17], Hochreiter found that in fact, these gradient-based training methods suffer from either exploding or vanishing gradients in an RNN, thus leading to either oscillating weights or extremely long or even failed training.

The Long Short-Term Memory (LSTM) recurrent architecture was introduced in [18] as an alternative formulation of the traditional recurrent neural network. Because the error compounds within the RNN due to feedback loops, if the gradient becomes either very small or relatively large, the learning will either slow down significantly or stall entirely (i.e., the gradient 'vanishes'), or the learning completely diverges (i.e., the gradient 'explodes'). Hochreiter and Schmidhuber [18] devised an architecture to allow for a 'constant error flow' through the RNN by introducing special, self-connected units called a memory cell. Each of these memory cells has four components: a self-connected neuron, an input gate, an output gate, and a forget gate. These gates control the information flow within the RNN and enable the network to bridge long time lags in the input. A schematic of a single memory cell is shown in Figure 4 The single memory cells are placed into the hidden layer. The equations to compute the memory cell output are as follows [18], starting with computations performed on a vector input, $\mathbf{y}(t-1)$, in the memory cell, $c_{j}$ :

$$
\begin{aligned}
\text { net }_{c_{j}}(t) & =\mathbf{w}_{c_{j}}^{T} \mathbf{y}(t-1) \\
\text { net }_{\text {in }_{j}}(t) & =\mathbf{w}_{i_{j}}^{T} \mathbf{y}(t-1) \\
\operatorname{net}_{f_{j}}(t) & =\mathbf{w}_{f_{j}}^{T} \mathbf{y}(t-1) \\
\text { net }_{\text {out }_{j}}(t) & =\mathbf{w}_{\text {out }_{j}}^{T} \mathbf{y}(t-1)
\end{aligned}
$$

where $\mathbf{w}_{c_{j}}, \mathbf{w}_{i n_{j}}, \mathbf{w}_{f_{j}}$, and $\mathbf{w}_{\text {out }_{j}}$ are vectors of weights that are determined during LSTM ANN training, and ${ }^{T}$ denotes the transpose. These intermediate computations are then each passed through a 'squashing function" such as a sigmoid to obtain the 
activation at time $t$ for the "input gate":

the activation at time $t$ for the "forget gate":

$$
y_{i n_{j}}(t)=f_{i n_{j}}\left(\text { net }_{i n_{j}}(t)\right)
$$

$$
y_{\text {forget }_{j}}(t)=f_{\text {forget }_{j}}\left(\text { net }_{f_{j}}(t)\right),
$$

which are then used to calculate the internal state of the memory cell, $s_{c_{j}}(t)$ :

$$
s_{c_{j}}(t)=y_{\text {forget }_{j}}(t) s_{c_{j}}(t-1)+y_{i n_{j}}(t) g\left(\text { net }_{c_{j}}(t)\right), \quad \text { for } t>0,
$$

and $s_{c_{j}}(0)=0$. The final memory cell output, $y_{c_{j}}(t)$ is then computed by multiplying (i.e., gating) it by the output gate activation, $y_{\text {out }_{j}}(t)$ :

$$
y_{c_{j}}(t)=y_{\text {out }_{j}}(t) h\left(s_{c_{j}}(t)\right)
$$

where $y_{\text {out }}(t)$ is computed similarly to the other gate outputs: $y_{\text {out }}$ ( $(t)=f_{\text {out }_{j}}\left(\right.$ net $\left._{\text {out }}(t)\right)$.

The authors who developed the LSTM architecture note from their experiments that the LSTM appears to work well over a wide range of parameters, thus reducing the need for fine parameter tuning. Since its inception, the LSTM has been widely adopted amongst the machine learning community in state-of-the-art pattern recognition tools. It is for these reasons that the LSTM will be employed in this work to investigate the predictability of the ventilation data.

\section{2 | Applying LSTM RNNs to Ventilation Parameter Prediction}

The general LSTM network used in this work can be conceptualized as shown in Figure 2 but with the memory cells depicted in Figure 4 with feedback replacing each node in the hidden layer. Two experiments were conducted using a single hidden layer to predict a ventilation metric at time $t+1$ and time $t+10$, and an additional experiment was conducted where another hidden layer was added (i.e., stacked) to again try and predict at time $t+10$, where $t$ is the current time. Within the collected ventilation data, the Minute Ventilation measure is of particular interest since it is indicative of how well the mechanical ventilation is performing. For each of the four different ventilation modes, an LSTM network was trained to predict one time step ahead ( 1.5 seconds), using the measurements collected from the previous 5 time steps. The inputs consisted of multiple ventilation parameters, namely the spontaneous minute ventilation, expired tidal volume, and dynamic lung compliance.

\section{4 | RESULTS AND DISCUSSION}

Figures 5 and 6 show the resulting prediction as compared to the actual ventilation parameter, as tested on a separate, held-out ventilation session (i.e., the test data was not included in training the LTSM). The legend of each plot also displays the rootmean-squared (rms) error between the prediction and the actual data. For each of the four modes, the LSTM network is able to predict the next time step with reasonable accuracy, thus showing promise for the LSTM in ventilation response prediction.

However, the utility of being able to predict 1.5 seconds is minimal; it is really not enough time for any sort of meaningful action to take place should one be required. Furthermore, the broader vision for this work necessitates a farther view into the future. The range of prediction was therefore expanded to 15 seconds (i.e., 10 time steps), using data collected from the previous 15 seconds of ventilation. The results for each of the modes are shown in the top plots of Figures 7 to 10 , but as one may expect, yielded worse results than predicting 1.5 seconds ahead. The rms error increased by almost as much as 6 times that of the onetime-step prediction, and the predicted paths also appear shifted in time, indicating an inability to predict a much later time step given previous samples. Therefore, it is likely that some intermediate step is required. One more hidden layer was added to the LSTM network for each mode, where the first hidden layer was initially trained (i.e., pre-trained) to predict the data 5 time steps ahead by providing the actual data (from 5 time steps ahead) as the targeted outputs of the first layer, and then combined with the remaining layers to predict the data an additional 5 time steps ahead for a total of 10 time steps in the entire network. The idea behind the pre-training is to guide the intermediate layers towards the final desired result due to the increased model complexity introduced with additional hidden layers.

The results from using two hidden layers in this fashion is shown in the bottom plot of Figures 7 to 10 The addition of another hidden layer to facilitate intermediate predictions was successful in reducing the overall error in the network for a 15 second prediction into the future. It is noted, however, that the reduction in error for the SIMV modes are not as significant as the AC modes, but the AC modes exhibit nearly two to three times the amount of error than the SIMV modes when attempting to predict further in the future. 

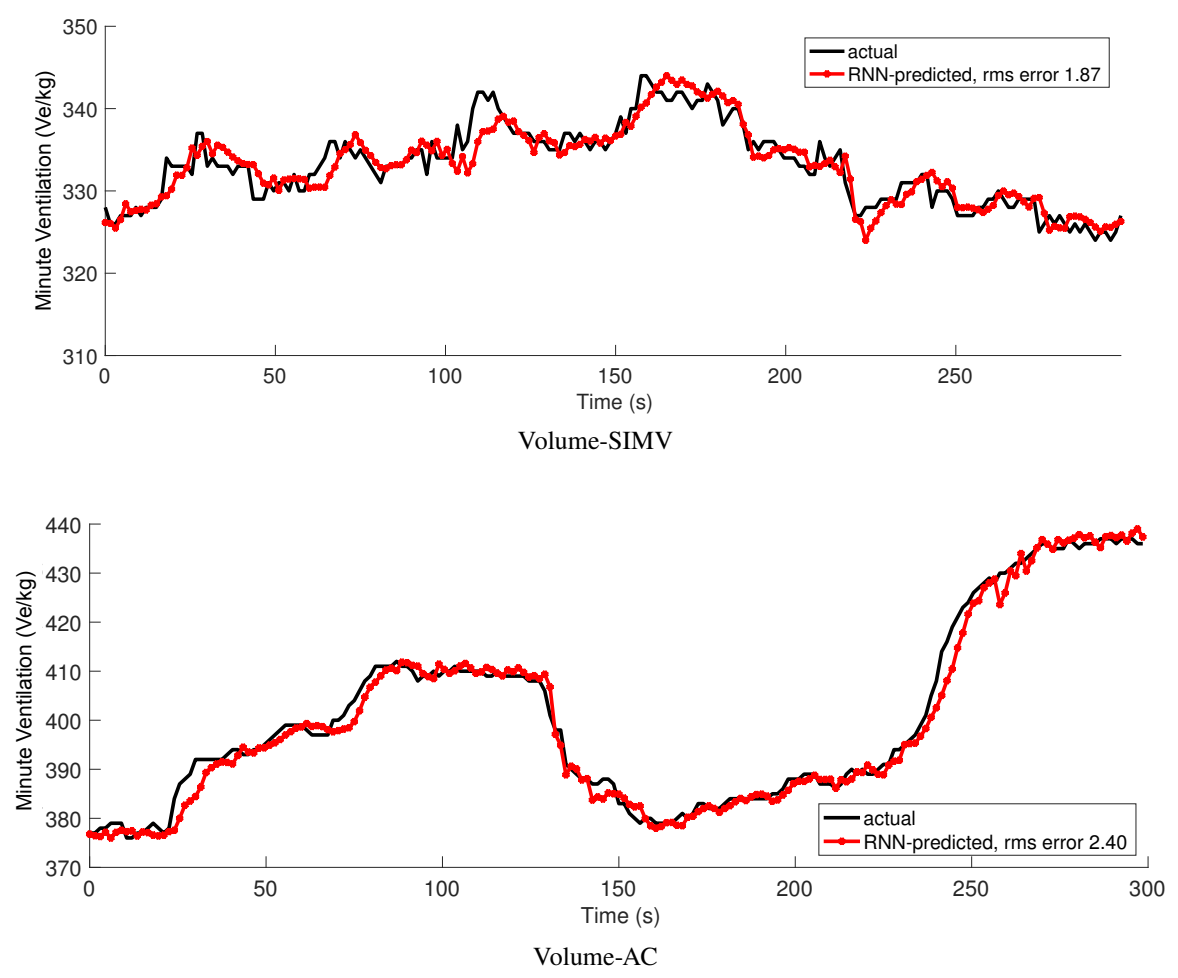

FIGURE 5 Prediction at time $t+1$ using the previous 5 samples in VCV mode.
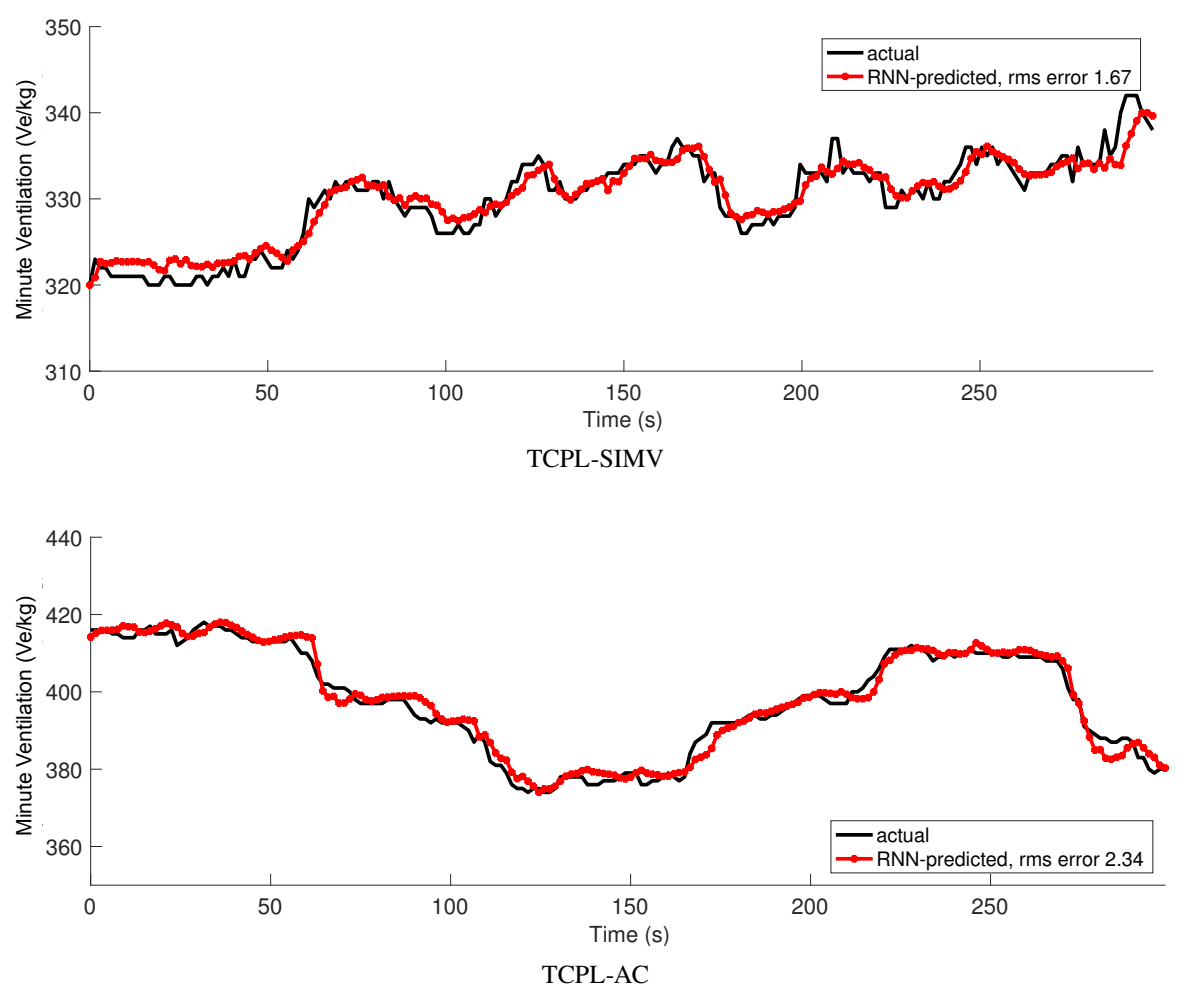

FIGURE 6 Prediction at time $t+1$ using the previous 5 samples in TCPLV mode. 

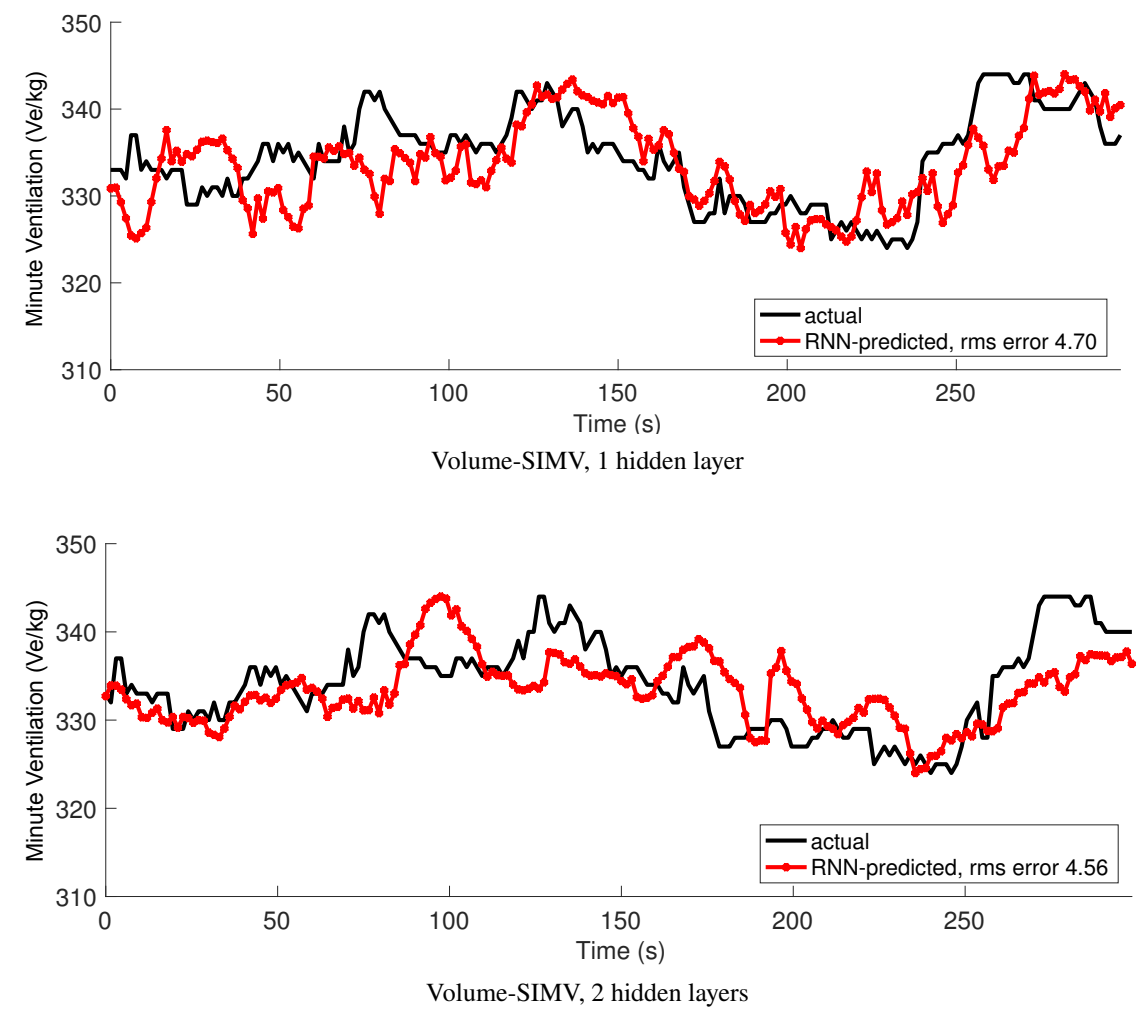

FIGURE 7 Prediction at time $t+10$ using samples from time $t-9$ to the current time, $t$, comparing one hidden layer to two hidden layers for the VCV-SIMV mode.

It is also noted that there is relatively large variation in the measurements over time, which the LSTM cannot always precisely predict. Prior work has shown the LSTM network to perform well applications such as speech recognition, handwriting recognition, and music onset detection, which generally do not require specific value predictions, and the efficacy of such a network on accurately tracking and predicting variable values has largely been unexplored. The predictions using the LSTM network appear to have difficulty in keeping up with frequent changes, but it may be that the trend of the ventilation response could be more important than maintaining a very close prediction to the exact value. Future work in this area will include consulting clinicians and doing further data analysis to determine the potential conditions under which a trend prediction would be acceptable or useful. Other methods can also be employed in increase the accuracy of the predictions, such as creating patient-specific models or characterization for prediction due to differing biologies (e.g., their breath patterns could differ, thus resulting in possible increased randomness in the response).

\section{5 | CONCLUSIONS AND FUTURE WORK}

It was shown in this work that applying machine learning approaches to ventilation data collected from preterm infants suffering from acute respiratory illness shows promise in its ability to predict future responses (i.e., the efficacy) of different mechanical ventilation modes. Towards improving care and treatment of preterm newborns, further development of this prediction process for ventilation could potentially aid in important clinical decisions or studies to improve preterm infant health. . In particular, the most logical outcome of the analysis used for this work is to aid in the development of real-time ventilation control systems that would be able to adjust parameters, or even change modes in extreme cases, to improve ventilation response. It is also expected that incorporating additional information regarding individual infants (e.g., birth weight, age, gender) may lead to better predictive models, since each infant is likely to respond differently to different modes of ventilation based on their biology. Future work in this area will involve additional data collection with this in mind, and a general framework for building infant-specific models will be developed, likely utilizing deeper networks and additional training techniques (e.g., dropout). 

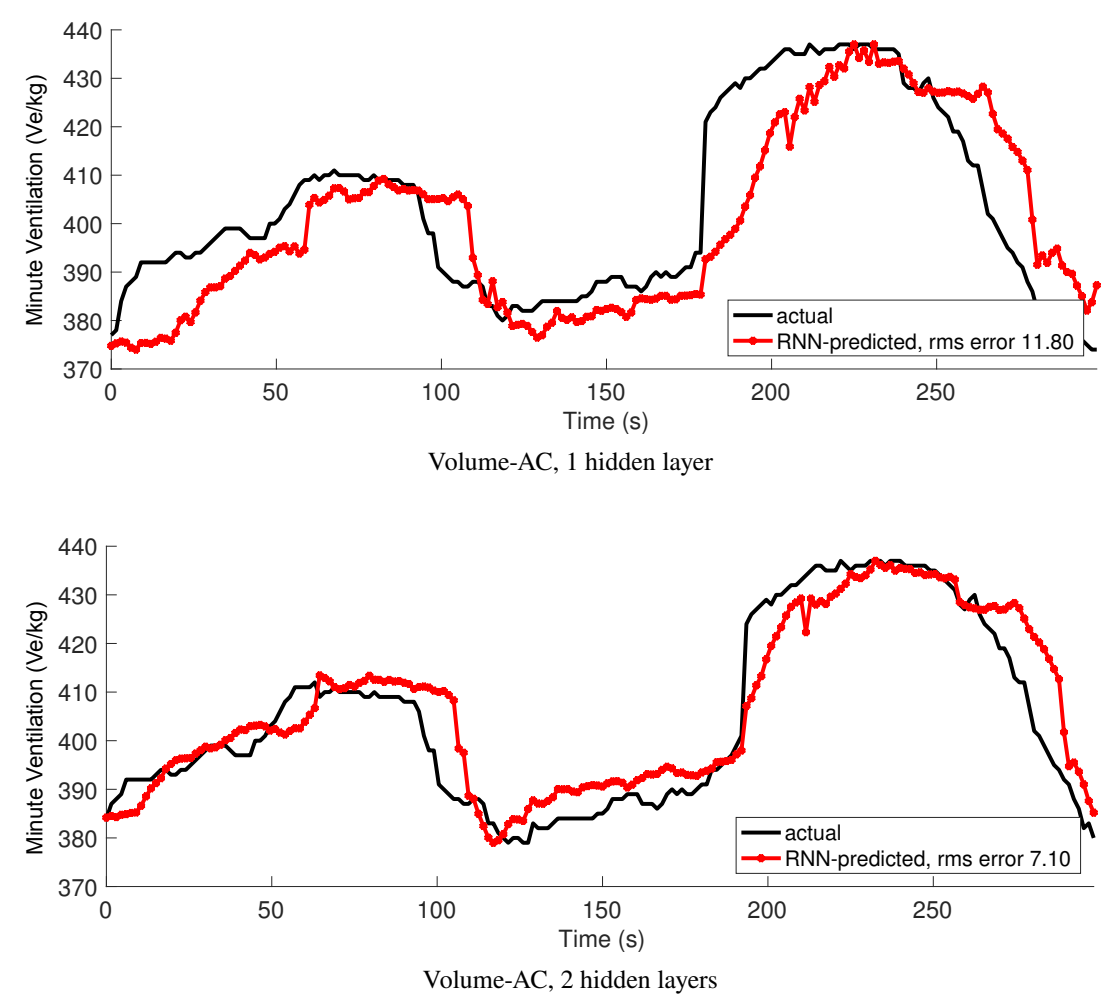

FIGURE 8 Prediction at time $t+10$ using samples from time $t-9$ to the current time, $t$, comparing one hidden layer to two hidden layers for the VCV-AC mode.
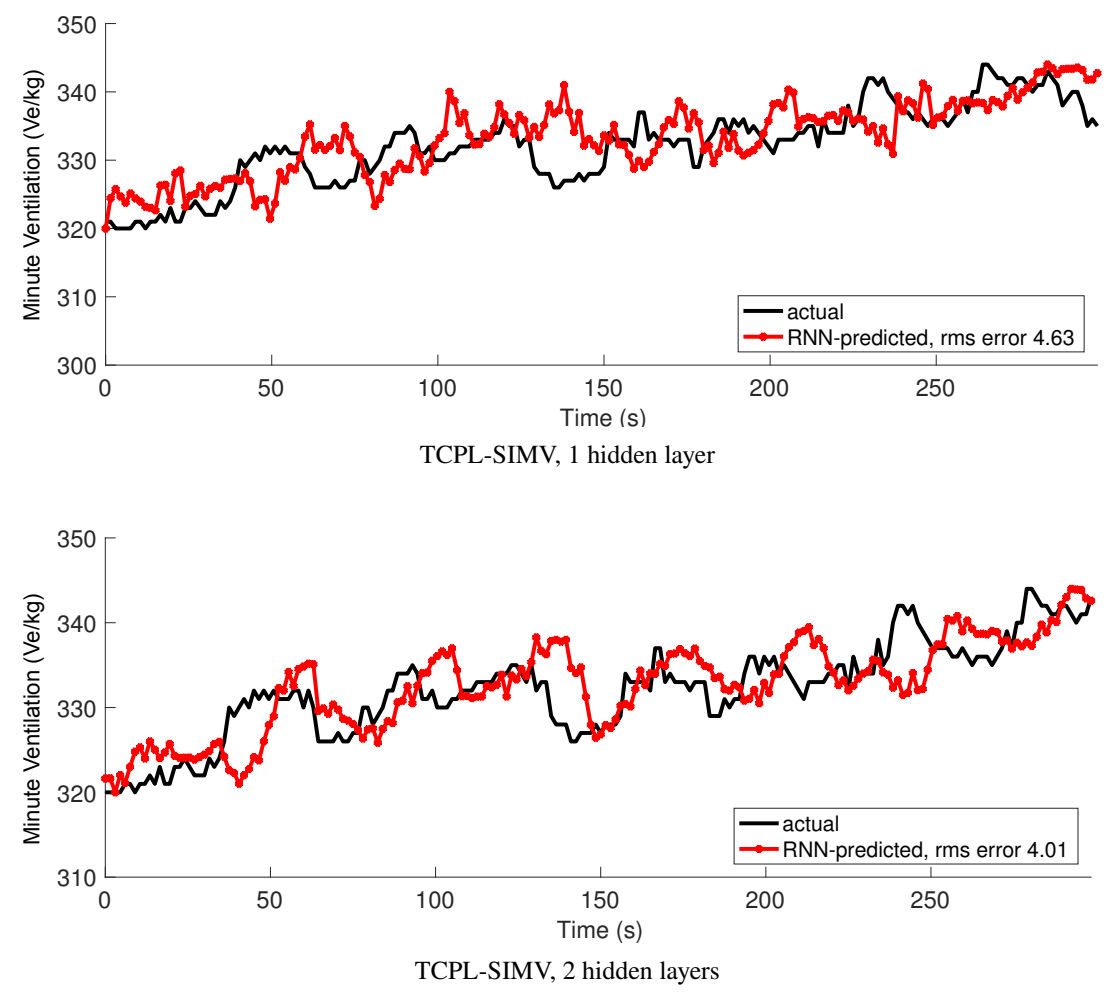

FIGURE 9 Prediction at time $t+10$ using samples from time $t-9$ to the current time, $t$, comparing one hidden layer to two hidden layers for the TCPLV-SIMV mode. 


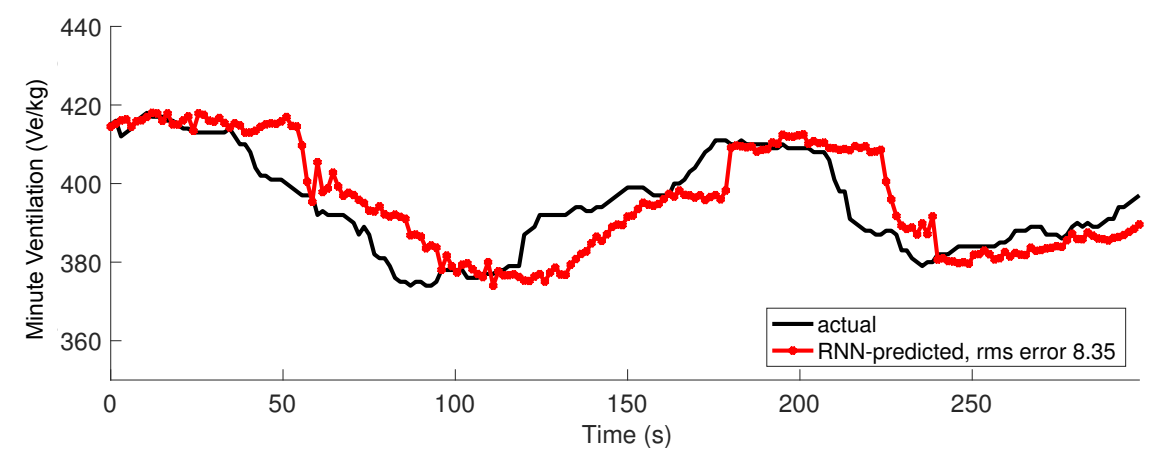

TCPL-AC, 1 hidden layer

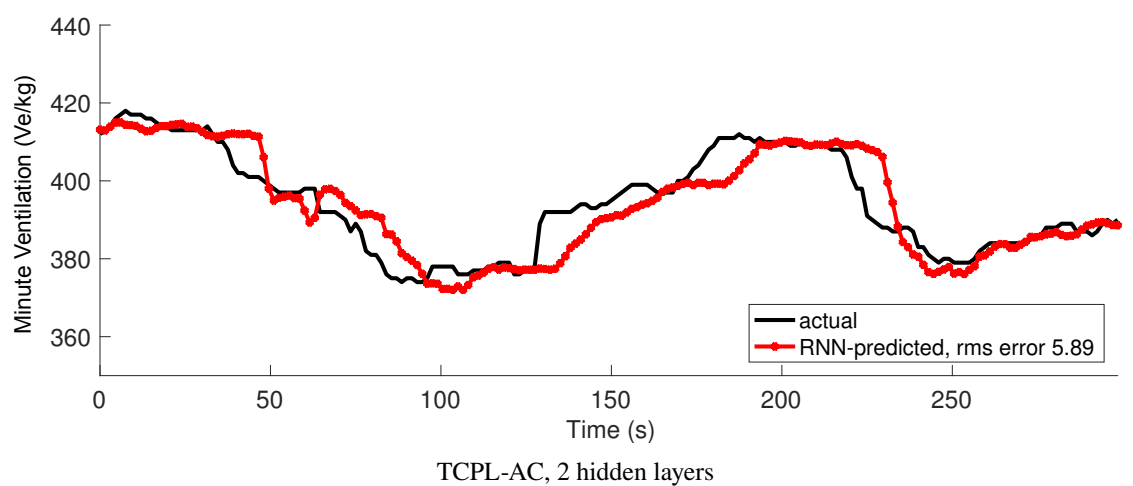

FIGURE 10 Prediction at time $t+10$ using samples from time $t-9$ to the current time, $t$, comparing one hidden layer to two hidden layers for the TCPLV-AC mode. 


\section{References}

[1] Sinha S.K., Gupta S., Donn S.M.. Immediate respiratory management of the preterm infant. Seminars in Fetal and Neonatal Medicine. 2008;13(1):24-29.

[2] Maulik U., Mukhopadhyay A., Chakraborty D.. Gene-Expression-Based Cancer Subtypes Prediction Through Feature Selection and Transductive SVM. IEEE Transactions on Biomedical Engineering. 2013;60(4):1111-1117.

[3] Mazzara G.P., Velthuizen R.P., Pearlman J.L., Greenberg H.M., Wagner H.. Brain tumor target volume determination for radiation treatment planning through automated MRI segmentation. International Journal of Radiation Oncology Biology Physics. 2004;59(1):300-312.

[4] Yoon J., Davtyan C., Schaar M.. Discovery and Clinical Decision Support for Personalized Healthcare. IEEE Journal of Biomedical and Health Informatics. 2017;21(4):1133-1145.

[5] Ball G., Aljabar P., Arichi T., et al. Machine-learning to characterise neonatal functional connectivity in the preterm brain. NeuroImage. 2016;124(Part A):267 - 275.

[6] Mani S., Ozdas A., Aliferis C., et al. Medical decision support using machine learning for early detection of late-onset neonatal sepsis. Journal of the American Medical Informatics Association. 2014;21(2):326-336.

[7] Shirwaikar Rudresh D., Acharya U. Dinesh, Makkithaya Krishnamoorthi, Surulivelrajan M., Lewis Leslie Edward Simon. Machine Learning Techniques for Neonatal Apnea Prediction. Journal of Artificial Intelligence. 2016;9:33-38.

[8] Mikhno A., Ennett C. M.. Prediction of extubation failure for neonates with respiratory distress syndrome using the MIMICII clinical database. 2012 Annual International Conference of the IEEE Engineering in Medicine and Biology Society. 2012;:5094-5097.

[9] Precup D., Robles-Rubio C.A., Brown K.A., et al. Prediction of extubation readiness in extreme preterm infants based on measures of cardiorespiratory variability. Engineering in Medicine and Biology Society (EMBC), 2012 Annual International Conference of the IEEE. 2012;:5630-5633.

[10] Ganzert S., Guttmann J., Kersting K., et al. Analysis of respiratory pressure-volume curves in intensive care medicine using inductive machine learning. Artificial intelligence in medicine. 2002;26(1):69-86.

[11] RuleQuest Research Pty Ltd. http://www.rulequest.com/ .

[12] Hornik Kurt. Approximation capabilities of multilayer feedforward networks. Neural Networks. 1991;4(2):251-257.

[13] Martens J., Sutskever I.. Learning recurrent neural networks with hessian-free optimization. In: :1033-1040; 2011.

[14] Williams R. J., Zipser D.. Gradient-based learning algorithms for recurrent networks and their computational complexity. Back-propagation: Theory, Architectures and Applications. 1992;1:322-486.

[15] Robinson A. J, Fallside F. The utility drive dynamic error propagation network. : Cambridge University Engineering Department; 1987.

[16] Y. Bengio P. Simard, Frasconi P.. Learning long-term dependencies with gradient descent is difficult. IEEE transactions on neural networks. 1994;.

[17] Hochreiter J.. Untersuchungen zu dynamischen neuronalen Netzen. PhD thesisTechnische Universitat Munchen1991.

[18] Hochreiter S., Schmidhuber J.. Long short-term memory. Neural Computation. 1997;9(8):1735-1780. 